\title{
Comparison of Polymerase Chain Reaction- Restriction Fragment Length Polymorphism (PCR-RFLP) and Repetitive Sequence-PCR (REP-PCR) for Differentiation of Riemerella anatipestifer Isolates
}

\author{
C. Devigasri $^{1 *}$, P. M. Priya ${ }^{1 *}$, M. Mini ${ }^{1}$, Surya Sankar ${ }^{1}$ and T. V. Aravindhakshan ${ }^{2}$ \\ ${ }^{1}$ Department of Veterinary Microbiology, College of Veterinary and Animal Sciences, \\ Mannuthy, Thrissur, Kerala, India \\ ${ }^{2}$ Department of Animal Breeding Genetics and Biostatistics, College of Veterinary and Animal \\ Sciences, Mannuthy, Kerala, India \\ *Corresponding author
}

\section{A B S T R A C T}

Keywords

Riemerella anatipestifer, PCRRFLP, REP-PCR, Electrophoretic patterns

Article Info

Accepted:

19 September 2019

Available Online:

10 October 2019
Riemerella anatipestifer, a pathogen causing infectious serositis and septicaemia in ducks, turkey, geese, pheasants, swans, poultry and also reported in pigs, which belongs to family Flavobacteriaceae. Twenty one serotypes have been reported so far by serum agglutination test which is found to be labour intensive and time consuming. In order to find out the better method to differentiate the isolates, PCR-RFLP and REP-PCR has been compared. The 20 isolates of $R$. anatipestifer were subjected to $16 \mathrm{~S}$ rRNA PCR and subjected to restriction digestion with Hae III and the banding pattern was observed for differentiation. On amplifying the repetitive extragenic palindromic sequences of $R$. anatipestifer, the electrophoretic patterns of PCR products obtained were considered for the classification. In both methods, the differences in banding pattern were analysed using PyElph 4.1 software for dendrogram construction. Both methods differentiated the isolates into three groups whereas REP-PCR was found to be the most efficient method owing to its easiness and repeatability. Hence, REP-PCR could be used for epidemiological investigation and characterisation of R.anatipestifer isolates.

\section{Introduction}

Riemerella anatipestifer (RA), is a Gram negative, microaerophillic, cocco-bacilli to short rods sometimes filamentous bacterium which belongs to the family Flavobacteriaceae, named to honour Rimer. It causes severe economic losses which mainly affects domestic ducks, turkey, geese, pheasants, swans, and also reported in pigs (Sandhu and Harry, 1981; Bisgaard, 1982;
Chang 1984; Sandhu and Leister, 1991; Loh et al., 1992). This disease is variously known as new duck disease, goose's flu, duck septicaemia, anatipestifer syndrome, riemerellosis, and infectious polyserositis (Chang et al., 2003; Christensen and Bisgaard 2009).

Twenty-one serotypes of $R$. anatipestifer have been reported worldwide. The occurrence of different serotypes in the field outbreaks have 
been reported which lacks cross protection between the isolates. (Harry 1969; Sandhu 1981; Bisgaard 1982; Layton and Sandhu, 1984; Loh 1992; Pathanasophon et al., 1996). The serotyping of isolates was based on raising antiserum against the isolates and also available only in reference laboratories which makes the method more cumbersome. In order to provide alternate method to antiserum phenotyping, molecular methods like PCRRFLP and REP-PCR have been tested to find out the genetic diversity.

The REP-PCR generates DNA fingerprints that discriminate bacterial strains based on distribution of repetitive extragenic sequences in prokaryotic genomes which is a tool for DNA-based epidemiologic assessment of clonal relationships among the bacterial isolates. The distance between the REP sequences varies among the bacterial strains which makes the difference in banding pattern (Woods et al., 1992). The PCR-RFLP, a technique which has been used by several researchers to detect polymorphism within a gene and such information has been useful for typing of the bacterial isolates.

The present study differentiated 20 isolates of $R$. anatipestifer based on banding pattern by RFLP obtained with HaeIII-digested 16S rDNA PCR, and also by REP-PCR patterns.

\section{Materials and Methods}

\section{Sample collection}

Samples for the isolation of $r$. anatipestifer were collected from ailing/dead birds brought to the Department of Veterinary Microbiology and Department of Veterinary Pathology, College of Veterinary and Animal Sciences, Mannuthy, for disease diagnosis. Heart blood, liver and spleen were collected aseptically and subjected for examination. Samples were also collected from sick/dead birds from University
Poultry and Duck Farm, Mannuthy. The isolates maintained in the Department of Veterinary Microbiology as preserved cultures in a lyophilised form were revived and also used in this study. A total of 20 isolates were analysed.

\section{Isolation and identification}

Samples were streaked on 5 to 10 per cent blood agar (BA) plate and incubated at $37^{\circ} \mathrm{C}$ for 12 to 24 hours micro aerobically for primary isolation. The lyophilised isolates were also revived using BA. To study the cultural characteristics, growth on Mac ConKey agar, haemolysis on BA were noted. To study the morphological characters, Gram's staining was done. The colonies suggestive of $R$. anatipestifer were subjected to conventional biochemical characterisation by means of oxidase, catalase, indole, gelatine liquefaction and ornithine decarboxylation (OIE, 2015; Surya et al., 2016).

\section{Identification by RA species- specific polymerase chain reaction}

Isolates were also identified by speciesspecific PCR assay by using primers designed by Kardos et al., (2007). (Fig. 1). Optimization of PCR was achieved through gradient PCR, by modification of annealing and extension temperatures. The amplification was checked by agarose gel electrophoresis. Five microliter of the PCR products was subjected to electrophoresis using one per cent agarose gel in $1 \mathrm{X}$ TBE buffer along with a molecular weight marker (100 bp) for relative sizing of PCR products.

\section{Serotyping}

The slide and tube agglutination tests were performed as per Sabnam (2015) using homologous and heterologous antisera raised against each isolates. 


\section{PCR- RFLP}

Primers based on the conserved region of the 16S rRNA gene designed by Pathnasophan et al., (2002) were used. Composition of single reaction mix for amplification is given in the table 1.

Optimization of PCR was achieved through gradient PCR, by modification of annealing and Extension temperatures. A Bio-Rad thermal cycler was pre- programmed for temperature and cycling conditions as given in the table 2 .

The PCR products were cooled to $4^{\circ} \mathrm{C}$ and stored at $-20^{\circ} \mathrm{C}$ for further characterisation.

\section{Restriction digestion}

The restriction digestion mixtures for Hae III were prepared as follows:

PCR product: $8 \mu \mathrm{L}$

Restriction enzyme: $1 \mu \mathrm{L}$

RE assay buffer: $2 \mu \mathrm{L}$

Nuclease free water: $4 \mu 1$

The tube containing reaction mixture was incubated at $37^{\circ} \mathrm{C}$ for 2 hours. The enzyme gets inactivated while adding loading buffer.

\section{Analysis of RE digests using Submarine agarose gel electrophoresis}

Based on the size, the restricted DNA fragments were separated using two per cent agarose gel in $1 \mathrm{X} \mathrm{TBE}$ at $50 \mathrm{~V} / \mathrm{cm}$ for $2 \mathrm{~h}$. By means of Gel Documentation System (BioRad, USA), gel images were compared and analysed.

\section{REP - PCR}

Published primers of Huang et al., (1999) were used and the composition of single reaction mix for amplification was depicted in the table 3 .

Optimisation of PCR was achieved through gradient PCR, by modification of annealing and extension temperatures. A Bio-Rad thermal cycler was pre- programmed for temperature and cycling conditions noted in the table 4 .

The PCR products were cooled to $4^{\circ} \mathrm{C}$ and stored at $-20^{\circ} \mathrm{C}$ till further analysis.

\section{Agarose gel electrophoresis of PCR product}

The amplification was checked by agarose gel electrophoresis. Twenty five microliter of the PCR products was subjected to electrophoresis using two per cent agarose gel in $1 \mathrm{X}$ TBE buffer along with a molecular weight marker (100 bp) for relative sizing of PCR products.

\section{Results and Discussion}

The 20 isolates were confirmed as $R$. anatipestifer conventional methods. On BA, colonies were smooth, convex, greyish-white, translucent and butyrous, measuring one to three millimeter in diameter was obtained. Similar colony characters were observed by Songer and Post (2005). All the isolates (RA1 to RA20) were Gram negative, non-motile and morphology varied from cocco-bacilli, short rods to filamentous forms, grew microaerobically and did not grow on MCA. They were non-haemolytic on BA.

A species-specific PCR assay developed by Kardos et al., (2007), revealed the presence of 546 bp PCR product. Similar results were observed by Soman et al., (2014) and Shancy, (2015) to confirm the RA isolates. This PCR assay, could easily replace the traditional method of identification to identify the isolate from clinical samples, as it was highly sensitive and specific. All the isolates used in 
this study were grouped under a single serotype by slide and tube agglutination tests.

Genomic DNA was extracted by phenol: chloroform method as suggested by Sambrook and Russell (2001) with few modifications. Molecular typing was carried out using PCRRFLP. The $R$. anatipestifer isolates (RA1 to RA20) were subjected to 16S rRNA PCR and the PCR products were subjected to restriction enzyme mediated digestion using Hae III. After suitable incubation, the digested products were electrophoresed in agarose gels. Five to ten bands were observed for all the isolates and differentiated based on the banding pattern. The isolates RA1, RA3, RA4, RA6, RA7, RA8, RA10, RA11, RA12 and RA15 showed the high intensity band approximately at $550 \mathrm{bp}$ and low intensity bands approximately at $120 \mathrm{bp}, 150 \mathrm{bp}, 250$ bp and $300 \mathrm{bp}$ were assigned into group a. The isolates RA2, RA9, RA13, RA14, RA16, and RA19 yielded distinct bands at $200 \mathrm{bp}, 270$ bp, $300 \mathrm{bp}$ and $320 \mathrm{bp}$, formed another groupb. Two of the isolates, RA17 and RA20 showed bands at $550 \mathrm{bp}, 500 \mathrm{bp}, 450 \mathrm{bp}$ and 250 bp were grouped into c. As RA5 and RA18 showed banding pattern with 90 per cent similarity to group $\mathrm{b}$, they were also placed under group (b). These patterns are depicted in figures 1 and figures 2 .

The distinct and prominent bands were scored for the analysis. Each lane was detected and the isolates were grouped based on the presence or absence of band on agarose gel. The distance matrices were calculated using the PyElph 1.4 fingerprint analysis software and this result was directly used to construct the phylogenetic relationships among the isolates by the neighbour-joining ( $\mathrm{NJ}$ ) method using the same software (Fig. 3).

The primers which amplified the repetitive extragenic palindromic sequences of chromosomal DNA were used and the banding patterns of PCR products were taken into consideration for molecular typing. The REPPCR distinguished 20 isolates of $R$. anatipestifer into three subtypes. Subtype 1 showed high intensity bands at $450 \mathrm{bp}$ and $1200 \mathrm{bp}$ and less intensity bands at $250 \mathrm{bp}$, $600 \mathrm{bp}, 700 \mathrm{bp}$ and $1000 \mathrm{bp}$ which included RA1, RA2, RA3, RA4, RA6, RA7, and RA8. The same pattern was showed by RA11 and RA15 except at 450 bp hence it was also included in subtype 1. Subtype 2 which included RA5, RA9 and RA10 showed clear high intensity bands at $400 \mathrm{bp}, 1000 \mathrm{bp}, 1200$ bp and 1300 bp. RA12, RA13 and RA14 also showed the same pattern except at $400 \mathrm{bp}$, hence included in subtype 2. Subtype 3 includes RA16, RA17, RA18, RA19 and RA20 as they showed bands at $450 \mathrm{bp}, 700 \mathrm{bp}$ and 1000 bp (Fig. 4 and Fig. 5).

The phylogenetic relationship among the isolates was analysed and dendrogram was constructed using PyElph 1.4 software by NJ method (Fig. 6).

The isolates belonging to single serotype could be further subtyped by using PCR-RFLP and REP-PCR based on the difference in banding pattern. The PCR-RFLP, a method first established by Grodzicker et al., (1974) has been used by several researchers to detect polymorphism within a gene and such information has been useful for typing of the isolates. A study conducted by Tsai et al., (2005) revealed the variations in the $16 \mathrm{~S}$ rRNA genes of $R$. anatipestifer. Similarly, Segers et al., (1993) and Subramaniam et al., (1997) reported that $16 \mathrm{~S}$ rRNA ( $r r s$ ) gene, has been successfully used in classifying $R$. anatipestifer as belonging to the Flavobacteriaceae in rRNA superfamily V. So, PCR- RFLP based on $16 \mathrm{~S}$ rRNA gene variation was used in this study. The variation in the banding pattern indicates the presence of variation in 16S rRNA gene among the isolates. 
Fig.1 Hae III digested RFLP pattern of RA1 to RA10

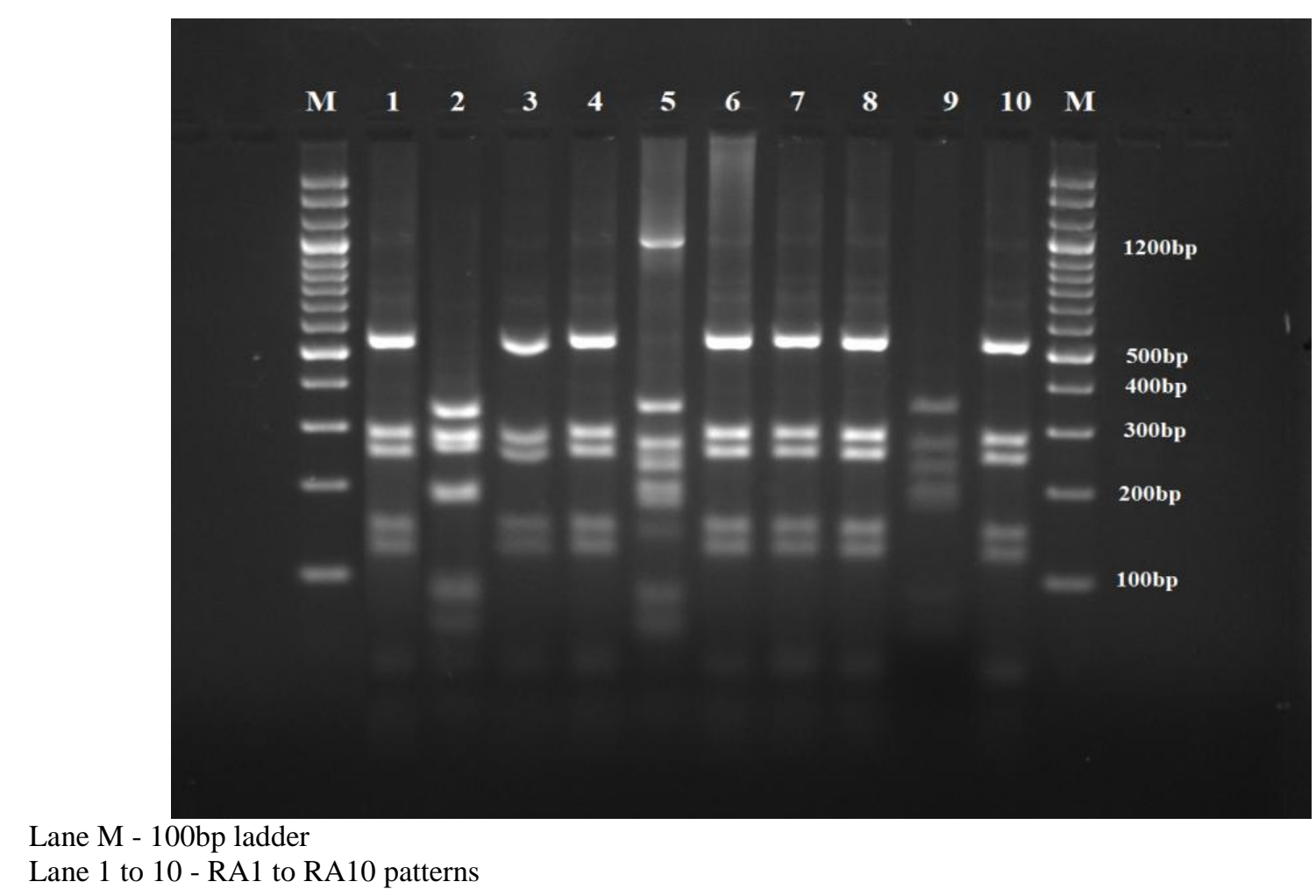

Fig.2 Hae III digested RFLP pattern of RA11 to RA20

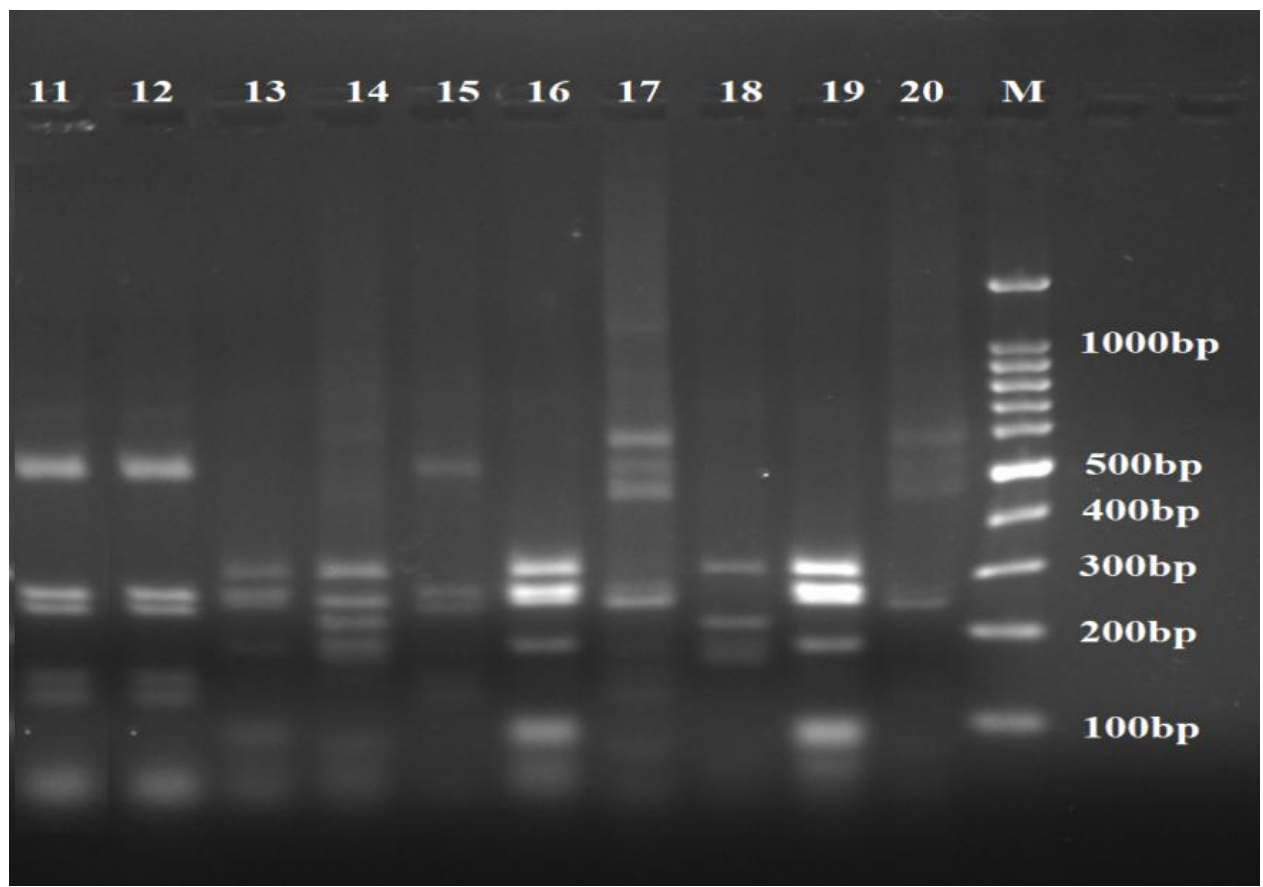

Lane $\mathrm{M}$ - 100bp ladder

Lane 11 to 20 - RA11 to RA20 patterns 
Fig.3 Phylogenetic analysis of RFLP profile using PyElph 4.1 with Neighbour joining method

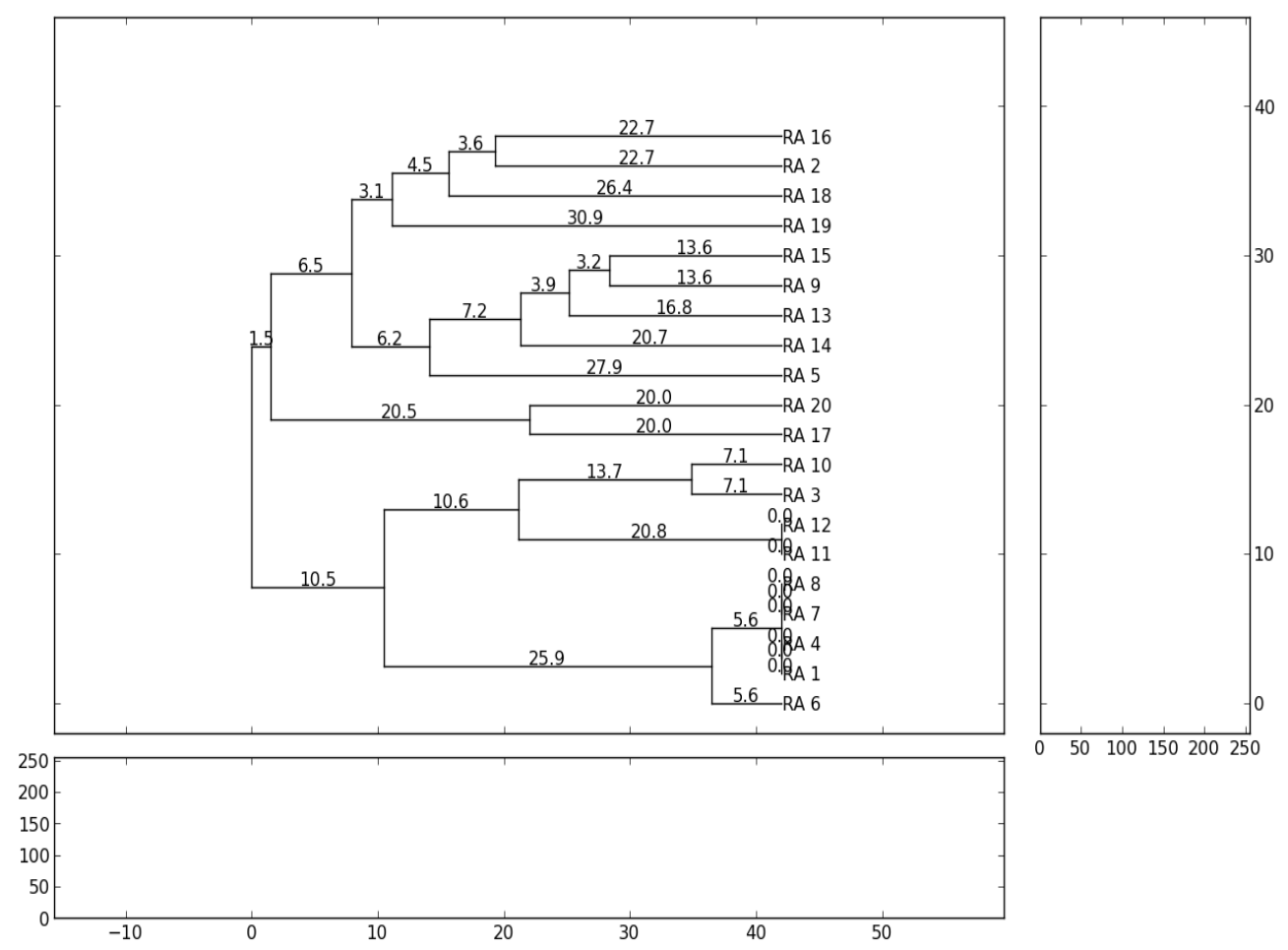

Fig.4 REP- PCR pattern for RA1 to RA10

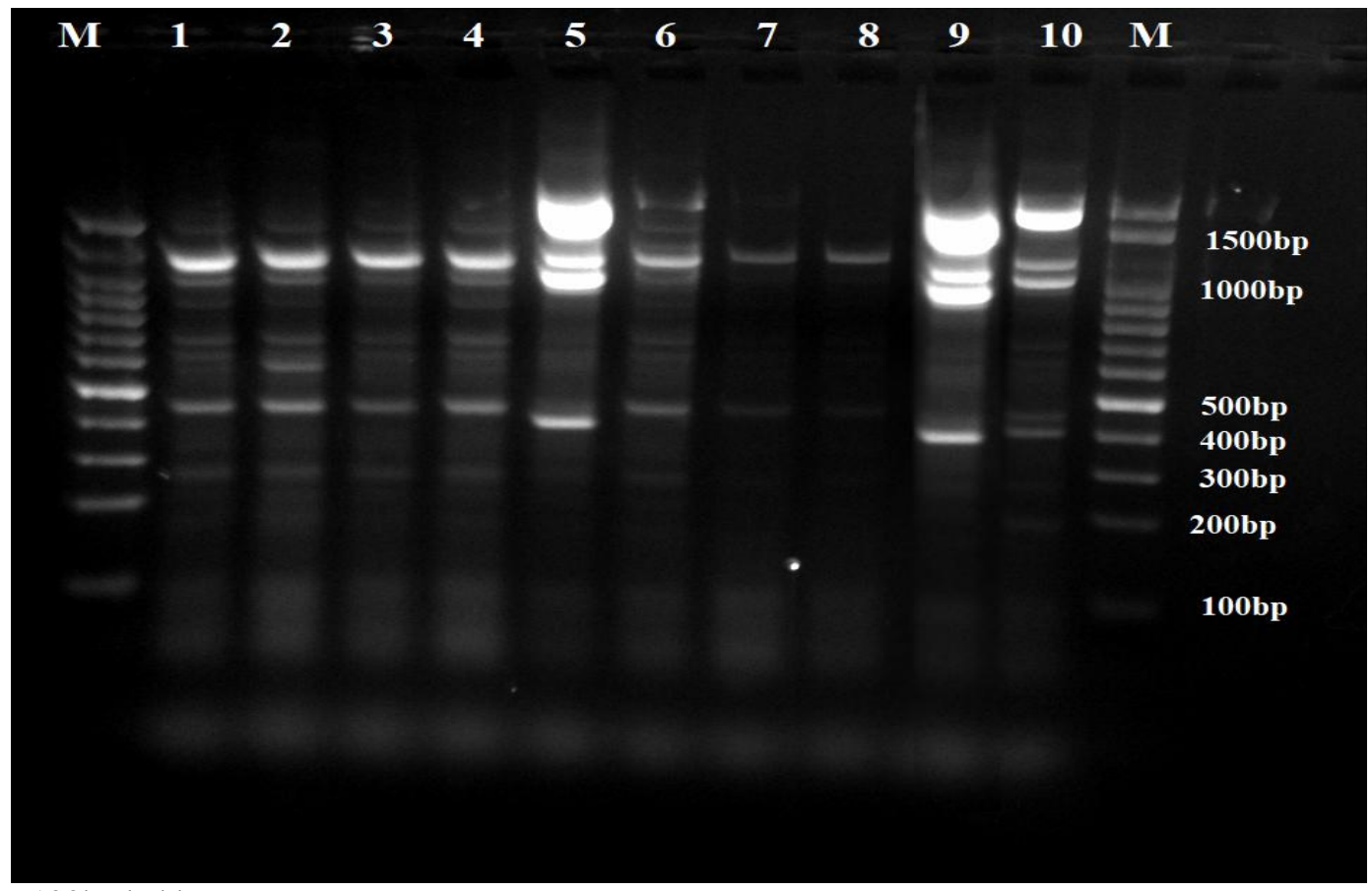

Lane $\mathrm{M}$ - 100bp ladder

Lane 1 to 10 - RA1 to RA10 banding patterns 
Fig.5 REP- PCR pattern for RA11 to RA20

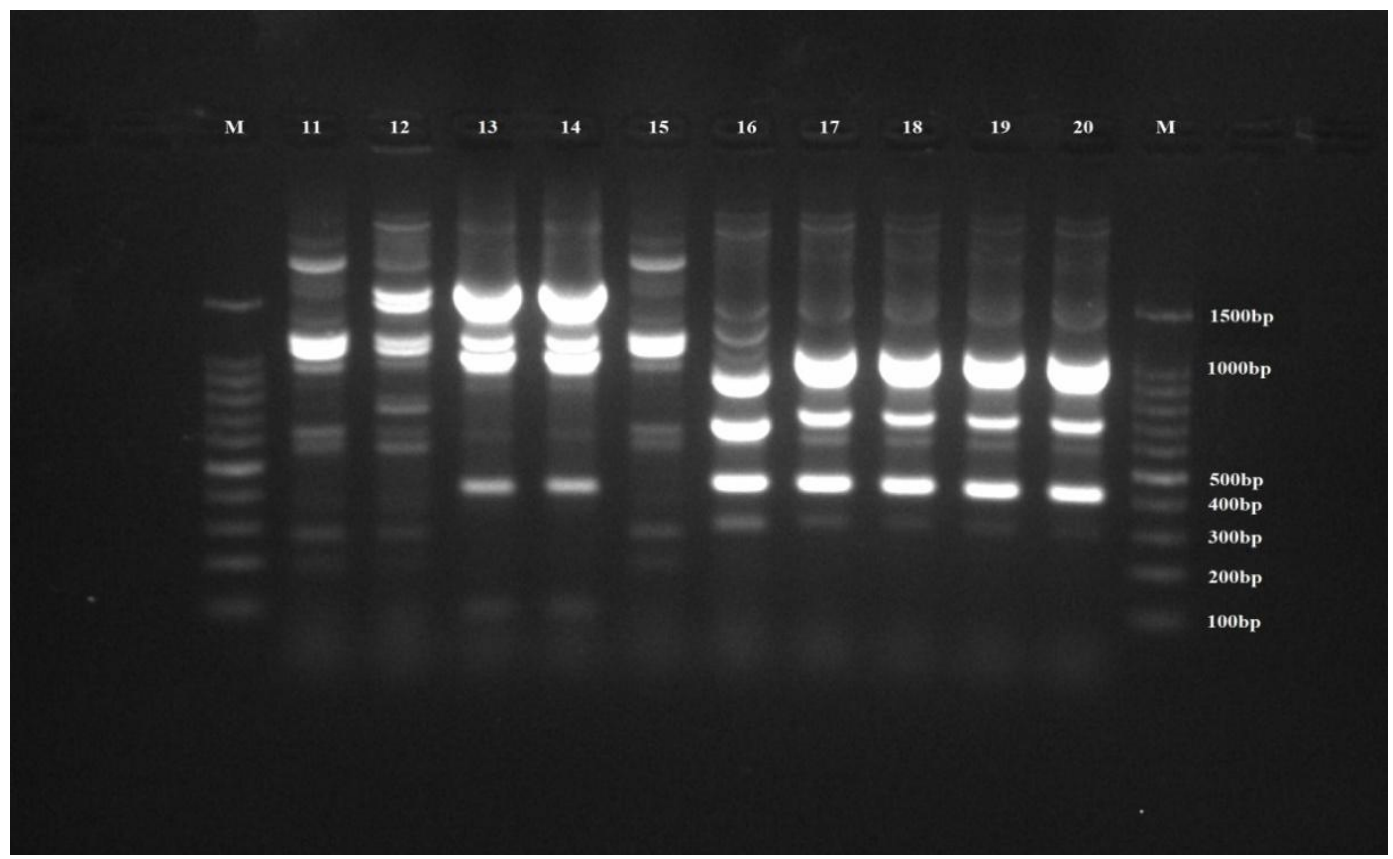

Lane $\mathrm{M}-100 \mathrm{bp}$ ladder

Lane 11 to 20 - RA11 to RA20 banding patterns

Fig.6 Phylogenetic analysis of REP- PCR patterns using PyElph 4.1 with Neighbour joining method (RA1 to RA20)

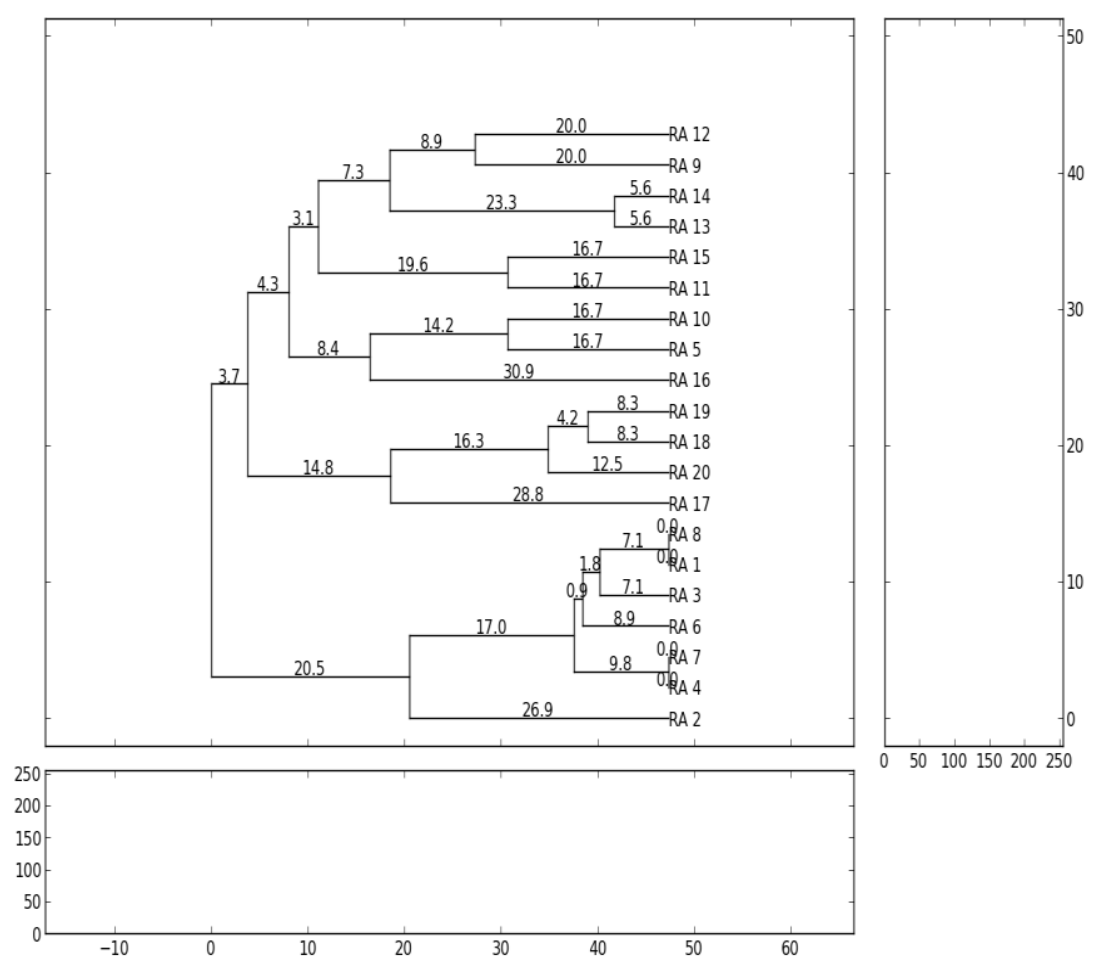


Table.1 Composition of PCR-RFLP

\begin{tabular}{|l|c|}
\hline EmeraldAmp® GT PCR Master Mix & $\mathbf{6 . 2 5} \boldsymbol{\mu L}$ \\
\hline Forward primer $(\mathbf{1 0} \mathbf{p M} / \boldsymbol{\mu L})$ & $1.0 \mu \mathrm{L}$ \\
\hline Reverse primer $(\mathbf{1 0} \mathbf{~ p M} / \boldsymbol{\mu L})$ & $1.0 \mu \mathrm{L}$ \\
\hline Template DNA $(\mathbf{5 0 n g} / \boldsymbol{\mu L})$ & $3.0 \mu \mathrm{L}$ \\
\hline Nuclease free water & $1.25 \mu \mathrm{L}$ \\
\hline Total volume & $12.5 \mu \mathrm{L}$ \\
\hline
\end{tabular}

Table.2 PCR programme for the amplification of $16 \mathrm{~S}$ rDNA sequence

\begin{tabular}{|l|l|}
\hline Initial denaturation & $95^{\circ} \mathrm{C}$ for $5 \mathrm{~min}$. \\
\hline Denaturation & $94^{\circ} \mathrm{C}$ for $30 \mathrm{sec}$. \\
\hline Annealing & $54^{\circ} \mathrm{C}$ for $50 \mathrm{sec}$. \\
\hline Extension & $72^{\circ} \mathrm{C}$ for $1 \mathrm{~min}$. \\
\hline Go to step 2 $(34$ times $)$ & \\
\hline Final extension & $72^{\circ} \mathrm{C}$ for $7 \mathrm{~min}$. \\
\hline
\end{tabular}

Table.3 Composition of PCR mastermix for REP-PCR

\begin{tabular}{|l|c|}
\hline EmeraldAmp® GT PCR Master Mix & $\mathbf{1 0 . 0} \boldsymbol{\mu L}$ \\
\hline Forward primer $(\mathbf{1 0} \mathbf{p M} / \boldsymbol{\mu L})$ & $5.0 \mu \mathrm{L}$ \\
\hline Reverse primer $(\mathbf{1 0} \mathbf{p M} / \boldsymbol{\mu L})$ & $5.0 \mu \mathrm{L}$ \\
\hline Template DNA $(\mathbf{5 0 n g} / \boldsymbol{\mu L})$ & $2.0 \mu \mathrm{L}$ \\
\hline Nuclease free water & $3.0 \mu \mathrm{L}$ \\
\hline Total volume & $25 \mu \mathrm{L}$ \\
\hline
\end{tabular}

Table.4 PCR programme for the amplification of repetitive sequence

\begin{tabular}{|l|c|}
\hline Initial denaturation & $95^{\circ} \mathrm{C}$ for $7 \mathrm{~min}$. \\
\hline Denaturation & $90^{\circ} \mathrm{C}$ for $30 \mathrm{sec}$ \\
\hline Annealing & $50.5^{\circ} \mathrm{C}$ for $60 \mathrm{sec}$. \\
\hline Extension & $65^{\circ} \mathrm{C}$ for $8 \mathrm{~min}$. \\
\hline Go to step $2(30$ times $)$ & \\
\hline Final extension & $65^{\circ} \mathrm{C}$ for $16 \mathrm{~min}$. \\
\hline
\end{tabular}

In this connection, Pathanasophon et al., (2002) also assessed the prevalence of $R$. anatipestifer in Thailand and reported the existence of a new serotype as confirmed by RFLP using Hae III restriction enzyme by amplifying the rrs gene. The PCR-RFLP differentiated the isolates into three different groups. The isolates RA1, RA3, RA4, RA6,
RA7, RA8, RA10, RA11, RA12 and RA15 showed the high intensity band approximately at $550 \mathrm{bp}$ and low intensity bands approximately at $120 \mathrm{bp}, 150 \mathrm{bp}, 250 \mathrm{bp}$ and $300 \mathrm{bp}$, were grouped together and named as a. These results are in accordance with Pathanasophon et al., (2002) who observed the same banding patterns for strains, ATCC 
11845, 1062/91 and 698/95. The isolates RA2, RA9, RA13, RA14, RA16 and RA19 formed into distinct bands at $200 \mathrm{bp}, 270 \mathrm{bp}$, $300 \mathrm{bp}$ and $320 \mathrm{bp}$ were grouped into $\mathrm{b}$. The RA17 and RA20 showed bands at 550 bp, 500 bp, $450 \mathrm{bp}$ and $250 \mathrm{bp}$, and were grouped into c. The RA5 and RA18 were placed in group $\mathrm{b}$, as they showed 90 per cent similarity in banding pattern with group $b$. The group a and $\mathrm{c}$ revealed common bands at 550 and 250 bp. Group b was clearly different from the others. Subramaniam et al., (1997) also observed the differences in $r r s$ gene of $R$. anatipestifer strains, within the same serotype and suggested that less than 15 base differences in the $16 \mathrm{~S}$ rRNA gene generally belong to the same species.

The distance matrices were calculated using the PyElph 1.4 fingerprint analysis software and this result was directly used to construct the phylogenetic relationships among the isolates by the NJ method using the same software. Pavel and Vasile (2012) developed this software which automatically extracts data from gel images, computes the molecular weights of the analysed molecules or fragments and compares DNA patterns which result from experiments with molecular genetic markers.

The region between the repetitive sequences in the genome of $R$. anatipestifer were amplified by REP-PCR using two 18 mer oligonuleotide primers derived from the consensus sequence of REP elements. The bands detected on the gels represent amplification products of DNA between adjacent repetitive elements. Differences in band sizes apparently represent polymorphism in the distances between repetitive sequence elements in different strains (Woods et al., 1992). Twenty isolates from different outbreaks gave three different REP-PCR patterns, revealing substantial genomic diversity among the isolates. It was interesting to note that there were three REP patterns for the same serotype. Hence, it would be an evidence for genetic variation even within the same serotype isolated from different outbreaks. Huang et al., (1999) also concluded that same serotype had more than one REP pattern while different serotypes shared the same pattern. The distance matrices were calculated using the PyElph 1.4 fingerprint analysis software and grouped the isolates into three patterns.

By both PCR-RFLP and REP-PCR, all the isolates were differentiated into three groups which confirmed the genomic diversity within the same serotype. These methods are easy and less time consuming when compared to serotyping. The genetic diversity within and between the serotypes should be considered during vaccine production. Compared with PCR-RFLP, REP-PCR offers the advantage of ease of performance and the availability of results within hours. Hence, it was concluded that REP-PCR could be served as a useful tool for subtyping of $R$. anatipestifer isolates for epidemiologic investigations.

\section{Acknowledgement}

The authors are thankful to the Dean, College of Veterinary and Animal Sciences, Mannuthy for providing necessary facilities to carry out this work.

\section{References}

Bisgaard, M. 1982. Antigenic studies on Pasteurella anatipestifer, species incertae sedis, using slide and tube agglutination. Avian Pathol. 11: 341-350.

Chang, C.F., Lin, W.H., Yeh, T.M., Chiang, T.S. and Chang, Y.F. 2003. Antimicrobial susceptibility of Riemerella anatipestifer isolated from ducks and the efficacy of ceftiofur treatment. J. Vet. Diag. Invest. 15: 26-29.

Christensen, H. and Bisgaard, M. 2010. Phylogenetic relationships of Riemerella anatipestifer serovars and related taxa and an evaluation of specific PCR tests reported for 
R. anatipestifer. J. Appl. Microbiol. 108: 1612-1619.

Harry, E. G. 1969. Pasteurella (Pfeifferella) anatipestifer serotypes isolated from cases of anatipestifer septicemia in ducks. Vet. Rec. 84: 673.

Huang, B., Subramaniam, S., Chua, K.L., Kwang, J., Loh, H., Frey, J. and Tan, H.M. 1999. Molecular fingerprinting of Riemerella anatipestifer by repetitive sequence PCR. Vet. Microbiol. 67: 213-219.

Kardos, G., Nagy, J., Antal, M., Bistyak, A., Tenk, M. and Kiss, I. 2007. Development of a novel PCR assay specific for Riemerella anatipestifer. Lett. Appl. Microbiol. 44: 145148.

Kardos, G., Nagy, J., Antal, M., Bistyak, A., Tenk, M. and Kiss, I. 2006. Development of a novel PCR assay specific for Riemerella anatipestifer. Lett. Appl. Microbiol. 44: 145148.

Layton, H.W. \& Sandhu, T.S. (1984). Protection of ducklings with a broth-grown Pasteurella anatipestifer bacterin. Avian Diseases, 28: 718-726.

Loh, H., Teo, T.P. and H.C. Tan. 1992. Serotypes of Pasteurella anatipestifer isolates from ducks in Singapore: a proposal of new serotypes. Avian Pathol. 21: 453-459.

OIE [Office International des Epizooties/ World Organization for Animal Health]. 2015. Manual of Diagnostic Tests and Vaccine for Terrestrial Animals 1: 524-530.

Pathanasophon, P., Phuektes, P., Tanticharoenyos, T., Narongsak, W. and Sawada, T. 2002. A potential new serotype of Riemerella anatipestifer isolated from ducks in Thailand. Avian Pathol. 31: 267-270.

Pathanasophon, P., Sawada, T., Pramoolsinsap, T. and Tanticharoenyos, T. 1996. Immunogenicity of Riemerella anatipestifer broth culture bacterin and cell-free culture filtrate in ducks. Avian Pathol. 25: 705-719

Sabnam, V.S. 2015. Serotyping and molecular characterisation of Riemerella anatipestifer. M.Sc dissertation, Kerala Veterinary and Animal Sciences University, Pookode, 75p.

Sabnam, V.S. 2015. Serotyping and molecular characterisation of Riemerella anatipestifer. M.Sc dissertation, Kerala Veterinary and Animal Sciences University, Pookode, 75p.

Sambrook, J. R. and Russell, D. 2001. Molecular Cloning: A Laboratory Manual. ( $4^{\text {th }} \mathrm{Ed}$.). Cold Spring Harbor Laboratory Press, New York, 1879p.

Sandhu, T.S. and Harry, E.G. 1981. Serotypes of Pasteurella anatipestifer from commercial white Pekin ducks in the United States. Avian Dis. 25: 497-502.

Sandhu, T.S. and Leister, M.L. 1991. Serotypes of Pasteurella anatipestifer isolates from poultry in different countries. Avian Pathol. 20: 233-239.

Soman, M., Nair S. R., Mini, M., Mani, B.K. and Joseph, S. 2014. Isolation and polymerase chain reaction-based identification of Riemerella anatipestifer from ducks in Kerala, India. Vet. World.7: 765-769.

Songer, J.E. and Post, K.W. (2005). Veterinary Microbiology: Bacterial and Fungal Agents of Animal Disease. Elsevier Saunders, Missouri. 434p.

Surya, P.S. 2011. Isolation and characterization of Riemerella anatipestifer from ducks. M.V.Sc thesis, Kerala Veterinary and Animal Sciences University, Pookode, 82p.

Woods, C. R., Versalovic, J., Koeuth, T., Lupski, J. R. 1992. Analysis of relationships among isolates of Citrobacter diversus by using DNA fingerprints generated by repetitive sequence -based primers in the polymerase chain reaction. J. Clin. Microbiol. 30: 29212929

\section{How to cite this article:}

Devigasri C., P. M. Priya, M. Mini, Surya Sankar and Aravindhakshan T. V. 2019. Comparison of Polymerase Chain Reaction- Restriction Fragment Length Polymorphism (PCR-RFLP) and Repetitive Sequence-PCR (REP-PCR) for Differentiation of Riemerella anatipestifer Isolates. Int.J.Curr.Microbiol.App.Sci. 8(10): 2703-2712. doi: https://doi.org/10.20546/ijcmas.2019.810.311 